NIST Technical Note 2098

\title{
Analysis of Iris Images in Nicholas Nixon: The Brown Sisters
}

James R. Matey

Patrick J. Grother

George W. Quinn

This publication is available free of charge from: https://doi.org/10.6028/NIST.TN.2098

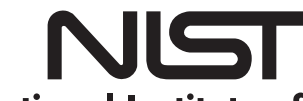

National Institute of Standards and Technology

U.S. Department of Commerce 


\title{
NIST Technical Note 2098
}

\section{Analysis of Iris Images in Nicholas Nixon: The Brown Sisters}

\author{
James R. Matey \\ Patrick J. Grother \\ George W. Quinn \\ Information Access Division \\ Information Technology Laboratory
}

This publication is available free of charge from:

https://doi.org/10.6028/NIST.TN.2098

June 2020

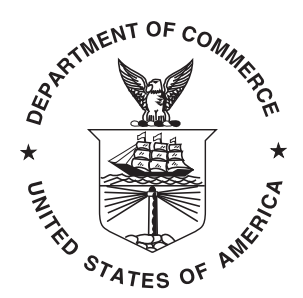

U.S. Department of Commerce

Wilbur L. Ross, Jr., Secretary

National Institute of Standards and Technology

Walter Copan, NIST Director and Undersecretary of Commerce for Standards and Technology 


\section{Disclaimers, Protocols and Licensing}

Certain commercial entities, equipment, software or materials may be identified in this document in order to describe an experimental procedure or concept adequately. Such identification is not intended to imply recommendation or endorsement by the National Institute of Standards and Technology (NIST), nor is it intended to imply that the entities, materials, software or equipment are necessarily the best available for the purpose.

All research reported in this paper based on data obtained from human subjects was carried out under protocols reviewed and approved by the NIST Research Protection Office (RPO) and/or the NIST Institutional Review Board. The NIST protocols relevant for this paper are:

- ITL-16-0035 Nixon Brown Sisters

- ITL-16-0026 IREX-IX

- ITL-2019-0156 IREX-X

All images shown in this paper have been checked for conformance to protocols/licensing regarding publication.

The high resolution Brown Sisters images used in the analyses described here are @Nicholas Nixon, and courtesy Fraenkel Gallery, San Francisco. The NIST license for those images does not permit redistribution/publication of the licensed images or analysis of the licensed images outside NIST's Biometric Research Laboratory. Readers with suggestions for additional analysis may contact the authors.

The examples of Nixon's images in this paper are low resolution images from the Fraenkel Gallery web pages and are @Nicholas Nixon, courtesy Fraenkel Gallery, San Francisco and used with permission.

National Institute of Standards and Technology Technical Note 2098

Natl. Inst. Stand. Technol. Tech. Note 2098, 21 pages (June 2020)

CODEN: NTNOEF

This publication is available free of charge from: https://doi.org/10.6028/NIST.TN.2098 


\begin{abstract}
In this paper we present results that expand on the Afghan Girl study by Daugman using gray-scale photographs of 4 subjects taken every year for 40 years: The Brown Sisters photographic project by Nicholas Nixon. We extracted iris images from the photographs and generated match scores using two commercial iris recognition algorithms. We developed criteria for making objective decisions about the utility of the extracted iris images and restricted the analysis of the mated pairs to those satisfying the criteria. The resulting match scores demonstrate matches at thresholds that correspond to FMR of 0.01 or better over periods of up to 9 years.
\end{abstract}

These are the initial results; optimization of the process may improve the results.

The Brown Sisters images used in this work are @Nicholas Nixon, and courtesy Fraenkel Gallery, San Francisco.

\title{
Key words
}

iris recognition; biometrics; Nicholas Nixon; Brown Sisters. 


\section{Table of Contents}

1 Introduction 1

2 Iris Image Extraction and Match Score Generation 1

3 Results \& Discussion $\quad 10$

4 Conclusions $\quad 13$

$\begin{array}{ll}\text { References } & 14\end{array}$

\section{List of Tables}

Table 1 Effects of Filters 


\section{List of Figures}

Fig. 1 The first picture (1975) in Nicholas Nixon's "The Brown Sisters" project. Low resolution example from the Fraenkel Gallery web page. This image is $\odot$ Nicholas Nixon, courtesy Fraenkel Gallery. Permission obtained.

Fig. 2 Illustration of the face extraction using the tool described in the text; the red stars indicate the locations of the annotations described in the text. Note the rotation to bring the eyes onto a horizontal line. The subject in the photo is a NIST employee.

Fig. 3 Illustration of the iris extraction using the tool described in the text. Note the rotation. The subject in the photo is a NIST employee.

Fig. 4 The 1992 picture in Nicholas Nixon's "The Brown Sisters" project; low resolution example from Fraenkel Gallery web page. Note lack of visible iris texture in the left and right-most subjects. This image is ONicholas Nixon, courtesy Fraenkel Gallery. Permission obtained.

Fig. 5 Cumulative distribution of the horizontal palpebral fissure for the 320 iris images (4 subjects, 2 eyes/subject) extracted from the 40 images described in the text. The cumulative distribution makes it easy to see what fraction is rejected as a function of the horizontal palpebral fissure.

Fig. 6 Simulation of a segmentation failure using an image from the IREX-X validation dataset[1]. Our license does not permit publication of the original high resolution Nixon images or extractions from those images.

Fig. 7 Distribution of the differences between the locations determined by the two algorithms for pupil centers.

Fig. 8 (Box-plot by algorithm and mated/non-mated of match scores converted to a common (FMR) scale.

Fig. 9 Plot of match score on FMR scale for mated images vs. time (years) between image collection by algorithm. 


\section{Introduction}

In the summer of 1975, Nicholas Nixon, a notable professional photographer, took a black and white photograph of his wife and her three sisters using a high quality, large format camera. That picture, see figure 1, is now iconic - as the first in a series of more than 40 photographs taken each year of the same four people, in the same left to right order, with high quality professional photography equipment. This series of images has been the subject of exhibitions at art galleries including MoMA ${ }^{1}$ and the Smithsonian ${ }^{2}$, and a book, Nicholas Nixon: The Brown Sisters. Forty Years[2].

Though not designed or collected for the purpose, these images present an interesting opportunity to test how well face and iris recognition algorithms work over a long (40 year) time span. NIST worked with Nixon's representative, the Fraenkel Gallery ${ }^{3}$, to obtain a license for use of high resolution digital scans of the 40 images available as of 2016 in biometric evaluations conducted at NIST. This paper is an initial analysis of the iris imagery extracted from those images; a separate analysis of the face imagery is in development.

\section{Iris Image Extraction and Match Score Generation}

Each of the forty images of the series was processed by a custom built image processing tool that enabled the operator to annotate the following locations on the faces of each of the subjects:

- Top of forehead

- Tip of nose

- Bottom of chin

- Left eye, medial and lateral canthi

- Right eye, medial and lateral canthi

- Left ear - left-most extent of the face

- Right ear - right-most extent of the face

The tool then used these annotations to extract faces and left/right eyes from the images

\footnotetext{
${ }^{1}$ Museum of Modern Art, New York, 11/22/2014-01/04/2015

${ }^{2}$ Smithsonian American Art Museum, Washington, March 2016 and online at americanart.si.edu/artist/ nicholas-nixon-3557

${ }^{3}$ Fraenkel Gallery, 49 Geary Street, 4th Floor, San Francisco, CA 94108; fraenkelgallery.com/gallery
} 


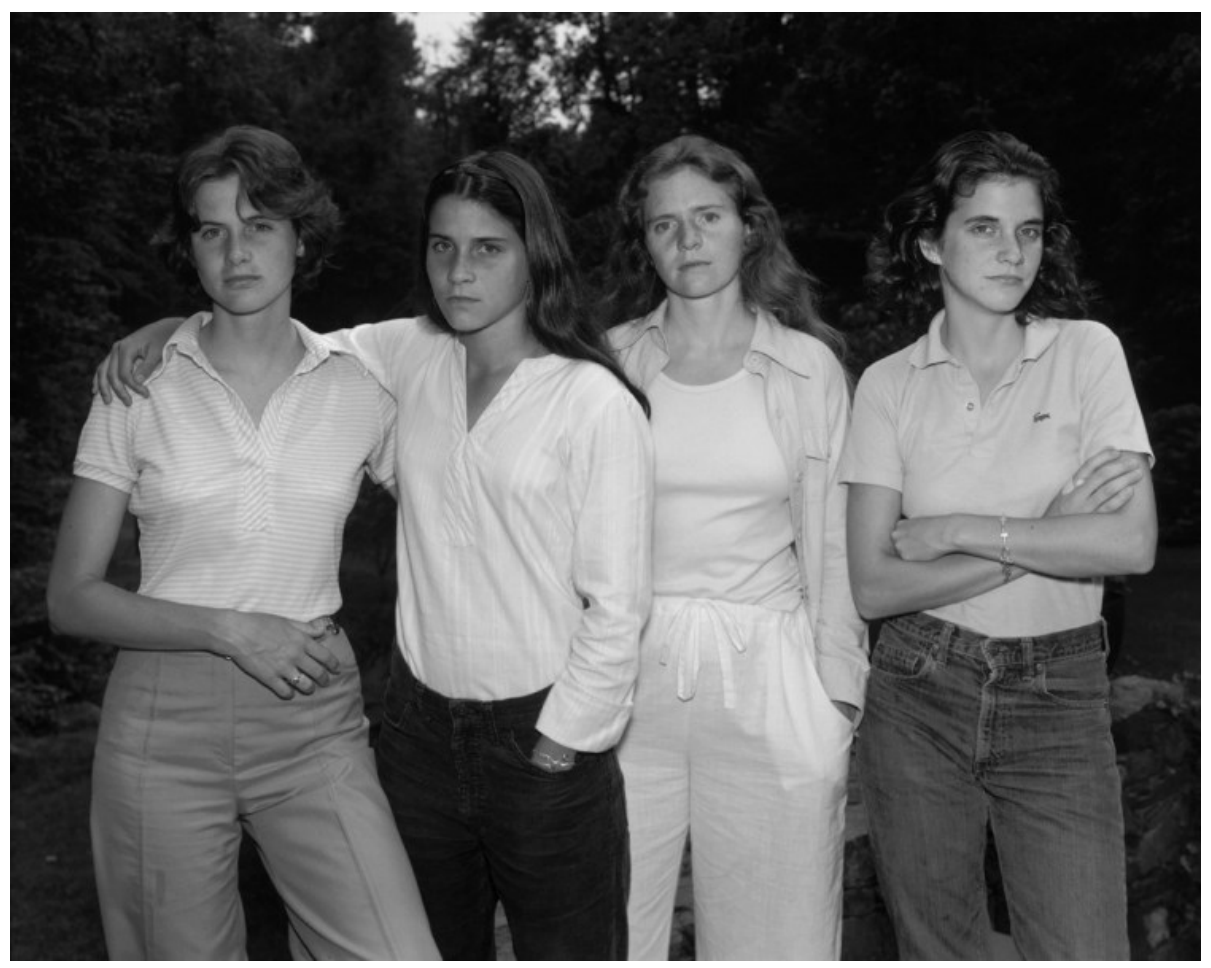

Fig. 1. The first picture (1975) in Nicholas Nixon's "The Brown Sisters" project. Low resolution example from the Fraenkel Gallery web page. This image is @Nicholas Nixon, courtesy Fraenkel Gallery. Permission obtained.

and store them as PNG files with lossless compression ${ }^{4}$, annotating the filenames with the year of the photograph, left/right for iris images and the left to right subject order. The extracted iris images were resized to $640 \times 480$ to assure compatibility with iris recognition algorithms; they were also rotated by the angle of a line between the pupils relative to the horizontal to mitigate the effects of side to side head pose (roll). We used the bilinear interpolation routines in .NET for these computations. Figures 2 and 3 illustrate the process using a high resolution image for which we have a model release for publication.

\footnotetext{
${ }^{4}$.NET Image.Save, see https://docs.microsoft.com/en-us/dotnet/api/system.drawing.image.save?view= netframework- 4.8
} 


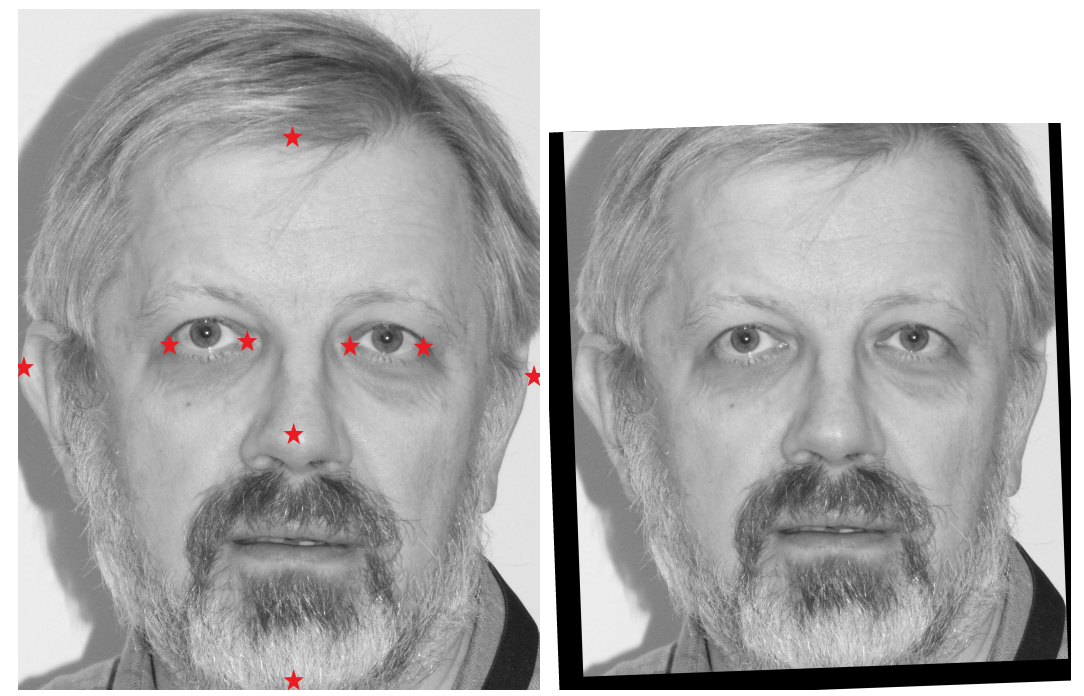

Fig. 2. Illustration of the face extraction using the tool described in the text; the red stars indicate the locations of the annotations described in the text. Note the rotation to bring the eyes onto a horizontal line. The subject in the photo is a NIST employee.

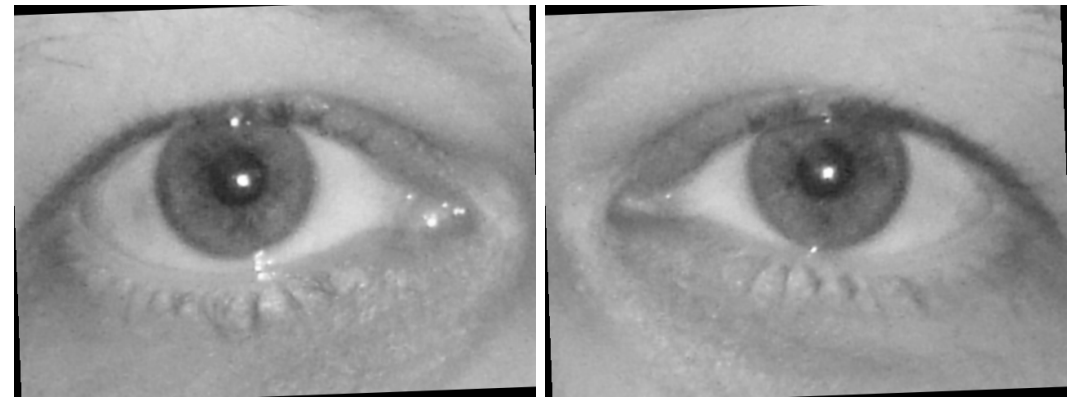

Fig. 3. Illustration of the iris extraction using the tool described in the text. Note the rotation. The subject in the photo is a NIST employee. 
Since the original images were taken with the goal of artistic aesthetics, rather than for the purpose of biometric investigation, there are extracted iris images which are not suitable for iris recognition due to problems including resolution, occlusion, shadowing, off axis effects, pose and specularities, as described in the IREX-V literature[3]. Figure 4 illustrates some cases where the iris texture is simply not visible in the original image.

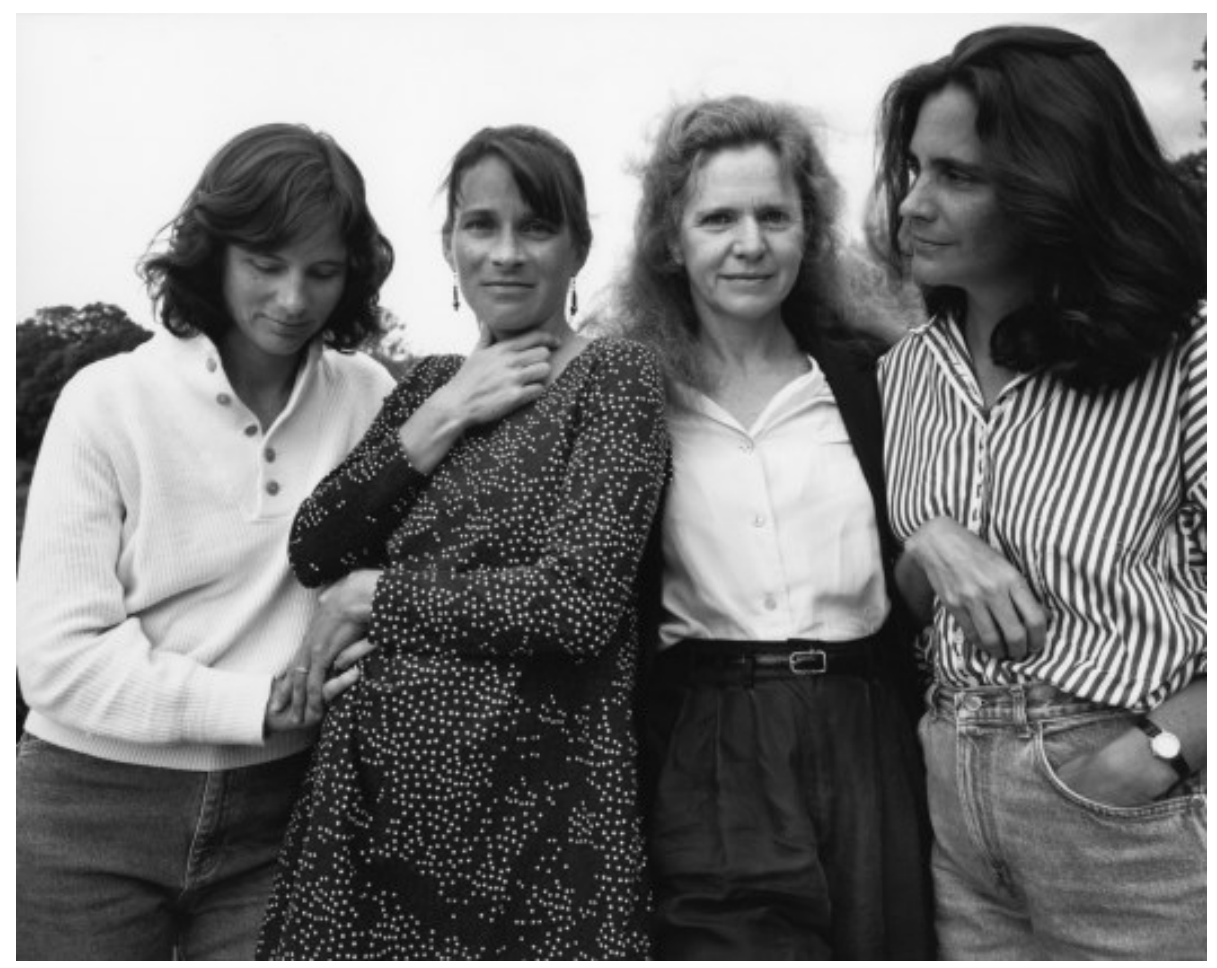

Fig. 4. The 1992 picture in Nicholas Nixon's "The Brown Sisters" project; low resolution example from Fraenkel Gallery web page. Note lack of visible iris texture in the left and right-most subjects. This image is @Nicholas Nixon, courtesy Fraenkel Gallery. Permission obtained.

An experienced image analyst could review such iris images and make subjective judgments on whether the images are of sufficient quality according to the IREX-V guidance. To avoid subjective judgments and to setup a process that could scale to a larger collection of imagery, we implemented a set of objective filters, as discussed below. Although we present the filters in an order in which they could be applied to the images, in practice, we processed all of the iris images to match scores, joined the iris image and template meta-data to the match score tables and applied the filters to the joined table.

The first of the filters is pixel resolution in the original image; we filter out iris images where the width of the iris, in pixels, in the original image is smaller than approxi- 
mately 45 pixels as discussed below. We base this criterion on results from IREX-IX[4] which showed a stable FNMR down to iris diameter of approximately 40 pixels ${ }^{5}$. We can compute the horizontal extent of the palpebral fissure, the distance between the medial and lateral canthi, for each iris, from the annotations described above. Since the medial and lateral canthi can be located even when eyes are closed, this metric is not dependent on whether the eyes are open, closed or partly open. The results for the 320 (40pictures $\times 4$ subjects $\times 2$ eyes) iris images are shown in the cumulative distribution plot in figure 5.

Since the ratio of the horizontal extent of the palpebral fissure to the diameter of the iris is about 2.2[5], and we want to leave some margin to allow for person to person variation, we drop all images for which the horizontal extent of the palpebral fissure in the original images is less than 100 pixels corresponding to an iris diameter of about 45 pixels. In future studies, sensitivity to the value of this criterion should be investigated. A value of 100 pixels removes approximately 160 of the 320 iris images from the analysis.

The iris images were then analyzed using a commercial-off-the-shelf (COTS) version of the iris2pi iris recognition algorithm whose internals have been described in detail by Daugman[6] [7] [8] [9] and variants of which have been implemented by academics, see for example Masek[10].

We also used another COTS algorithm, Neurotechnology/VeriEye Version 10, for which the internals are not well known.

These algorithms were chosen for this study because both of them provide diagnostic information that includes the centers of the pupil and iris as well as estimates of the quality of the images/resulting templates. With respect to the quality metric:

- For iris2pi, the quality metric is the number of bits set in the mask; the maximum value we have ever observed of that metric on outstanding quality images is approximately 1500; Daugman used 911 as a nominal value for his score normalization procedure[11]. For this analysis, we dropped images that could not produce at least 700 bits in the mask.

- The internal details of the quality metric provided by VeriEye are not known, we chose a minimum acceptable value of 50 in this analysis.

Any iris image that did not generate a template with a quality metric above these levels for either algorithm was discarded.

\footnotetext{
${ }^{5}$ This might be different for an algorithm that was tuned to work on images of lower resolution.
} 
Some images produced poor segmentation which could be seen in diagnostic images that resulted from plotting the iris and pupil boundaries determined by the algorithms on the iris images. We see a simulation of particularly poor segmentation in figure 6 . For an objective procedure, we computed the differences between the pupil centers determined by the two algorithms. The distribution of those differences can be seen in figure 7 . The secondary peak at 400 pixels is the result of failure of the segmentation process for one or both of the algorithms for a particular image, consistent with what was seen in the diagnostic images ${ }^{6}$. The simulation shown in figure 6 is a large failure; however, as shown by Matey et al.[12] even small errors in segmentation can lead to significant changes in Hamming distance for an iris2pi algorithm. Using an in silico simulation, they found a change in fractional Hamming distance of approximately 0.02 per pixel of pupil shift. They also presented a simple model of the effect which predicted 0.04 per pixel of shift. For this study, we discarded all images where the difference in either pupil or iris location between the two algorithms exceeded 5 pixels. In future studies, sensitivity to the value of this criterion should be investigated.

We are left with 18 out of the original 320 iris images after filtering; the effects of the various filters are shown in table 1.

Table 1. Effects of Filters

\section{Filter}

Horizontal extent palpebral fissure original image

Template generation success - iris2pi

Template generation success - VeriEye

Template generation success - both

Quality - iris2pi

Quality - VeriEye

Quality - both

Pupil centers agree

Iris centers agree

Pupil and iris centers agree

All filters satisfied (AND of all the above)
Iris Images Passing

166

206

288

190

134

189

99

81

107

57

18

This process does not deal explicitly with blur. It should. We plan to add an assessment of blur to our tool-set for future iris image evaluation. It is likely that manual intervention by an experienced image analyst could increase the number of iris images that satisfied all of the filters.

\footnotetext{
${ }^{6}$ Which we cannot reproduce here for licensing reasons.
} 


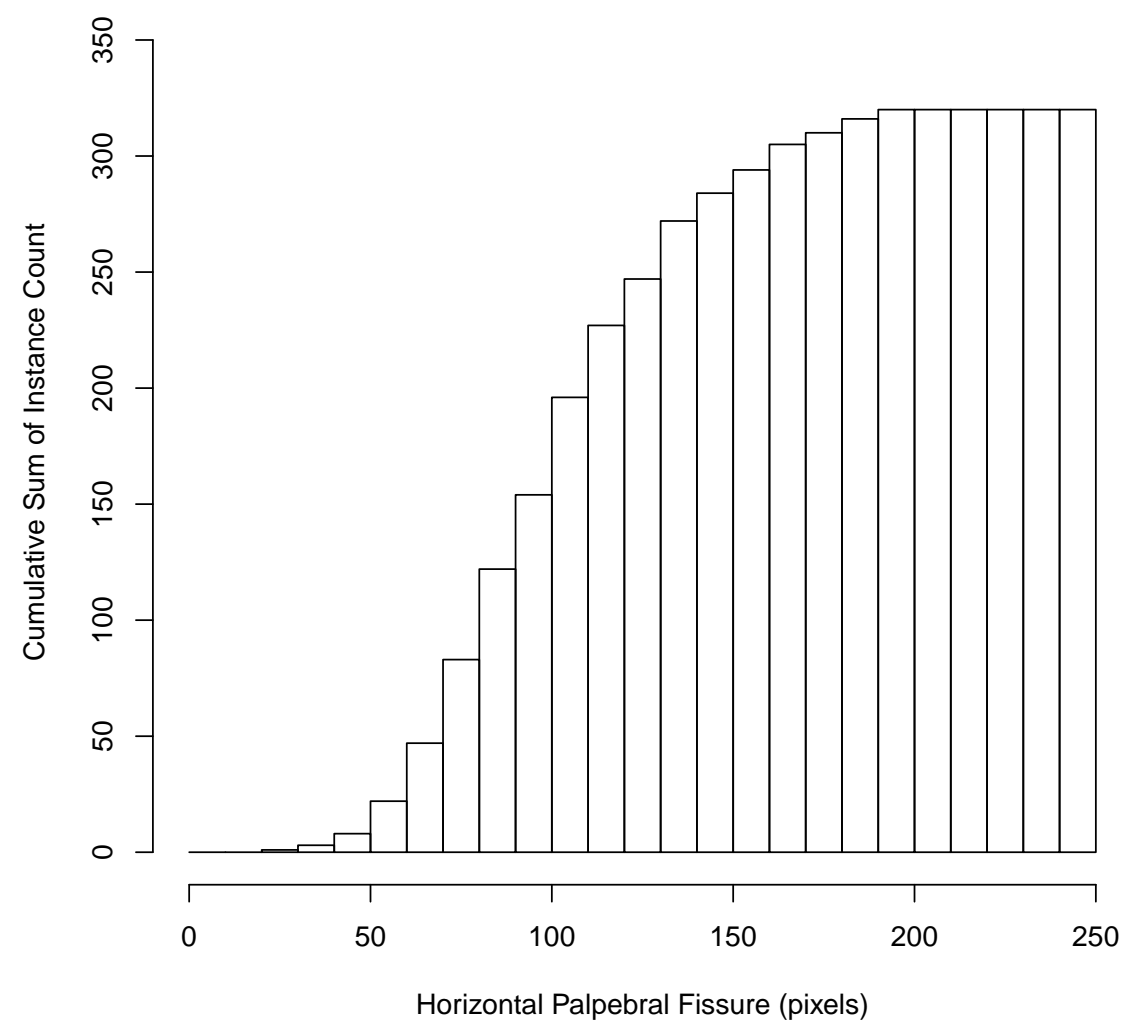

Fig. 5. Cumulative distribution of the horizontal palpebral fissure for the 320 iris images (4 subjects, 2 eyes/subject) extracted from the 40 images described in the text. The cumulative distribution makes it easy to see what fraction is rejected as a function of the horizontal palpebral fissure. 


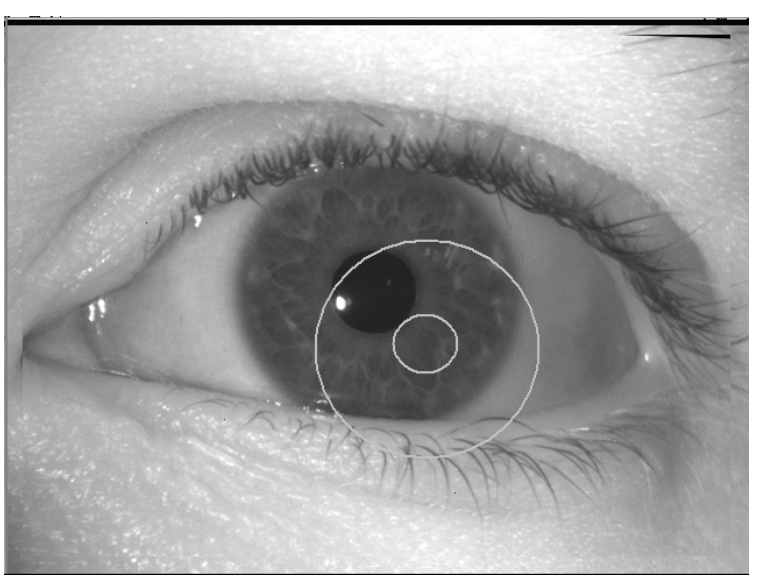

Fig. 6. Simulation of a segmentation failure using an image from the IREX-X validation dataset[1]. Our license does not permit publication of the original high resolution Nixon images or extractions from those images. 


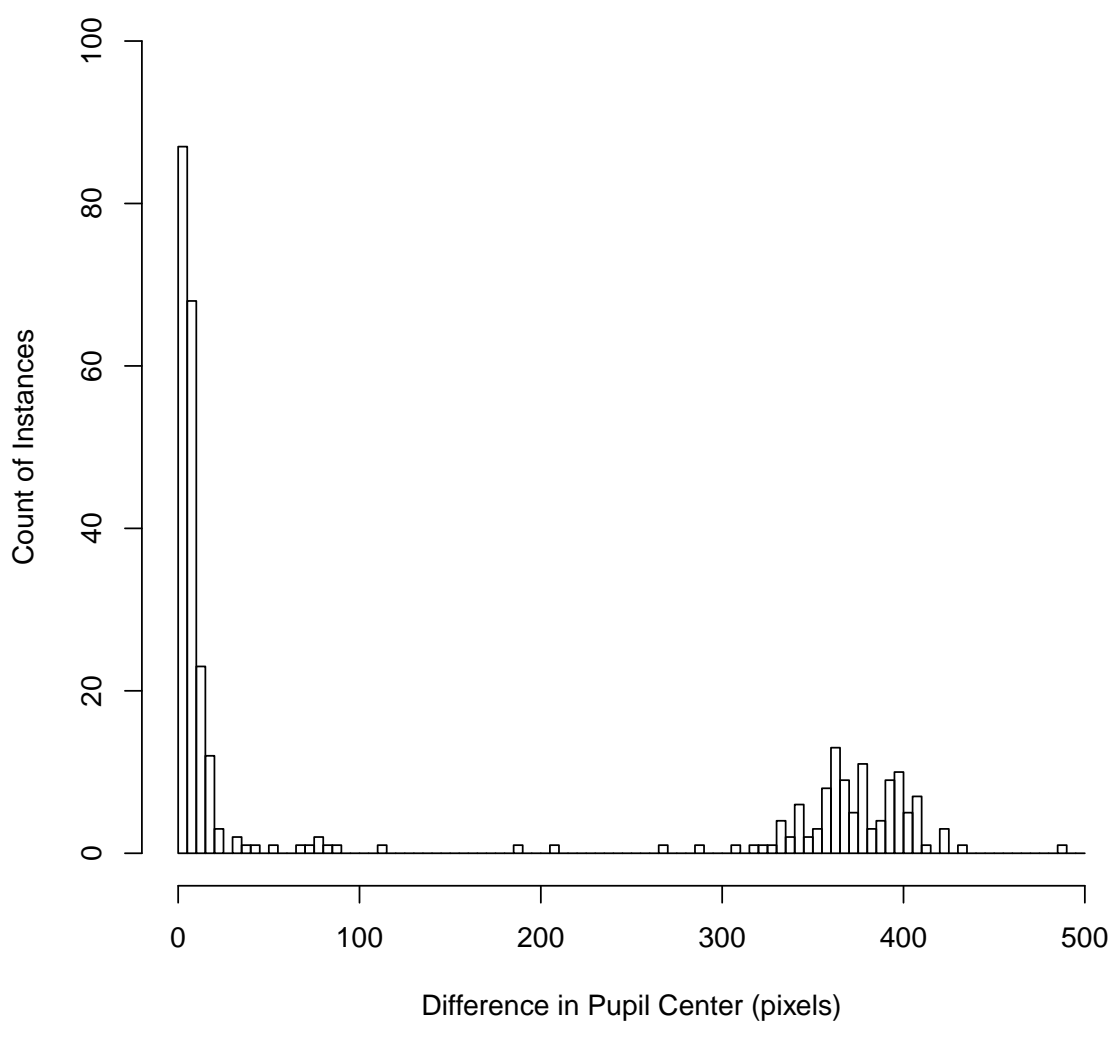

Fig. 7. Distribution of the differences between the locations determined by the two algorithms for pupil centers. 


\section{Results \& Discussion}

We then compared all the the templates for the remaining images for each of the algorithms and labeled the match scores as mated or non-mated based on the parameters (eye, subject) that labeled the iris images. We then converted the match scores to a common scale via the FMR vs score for the respective algorithms; the FMR vs score for iris2pi is based on Table 1 in Daugman's 2006 paper[8] and that for VeriEye on Section 5.2 of the Neurotechnology manual[13]. This provides a common scale for the two algorithms that can be more easily interpreted than raw match scores by the reader ${ }^{7}$. The results are seen in figure 8 .

The box-plot shows good separation between mated (Mated=TRUE) and non-mated (Mated=FALSE) for both algorithms. All of the mated images in this plot were at least one year apart. This suggests that analysis of iris images extracted from images which were not taken for the purpose of iris recognition can be used to determine if a person in one image is the same as a person in another image over time periods in excess of one year, provided that the extracted iris images conform to the filters described earlier. It might be possible to do better if the iris images were evaluated by an experienced analyst.

Figure 9 is a plot of mated scores on the FMR scale vs time delta between iris images for the data from figure 8 .

Figure 9 shows examples of strong matches out to 9 years. This is consistent with the results of IREX-VI[14], which showed "no evidence of a widespread iris ageing effect". We note that the Schuckers group at Clarkson[15] recently demonstrated that iris recognition is effective for children from the age of 4. Combining the Clarkson results with those in this paper we can infer that iris recognition may be useful in confirmation of the identity of people, including children, who have been missing for extended periods through the use of images that were not originally collected for the purpose of iris recognition. We are not the first to suggest this idea; an earlier demonstration spanning 15 years was reported by National Geographic[16] and documented by Daugman[17] for the case of the Afghan girl.

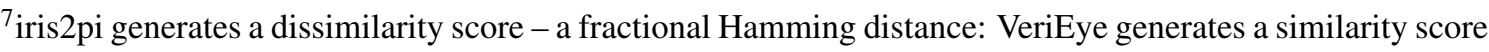
whose value is on an arbitrary, proprietary scale.
} 


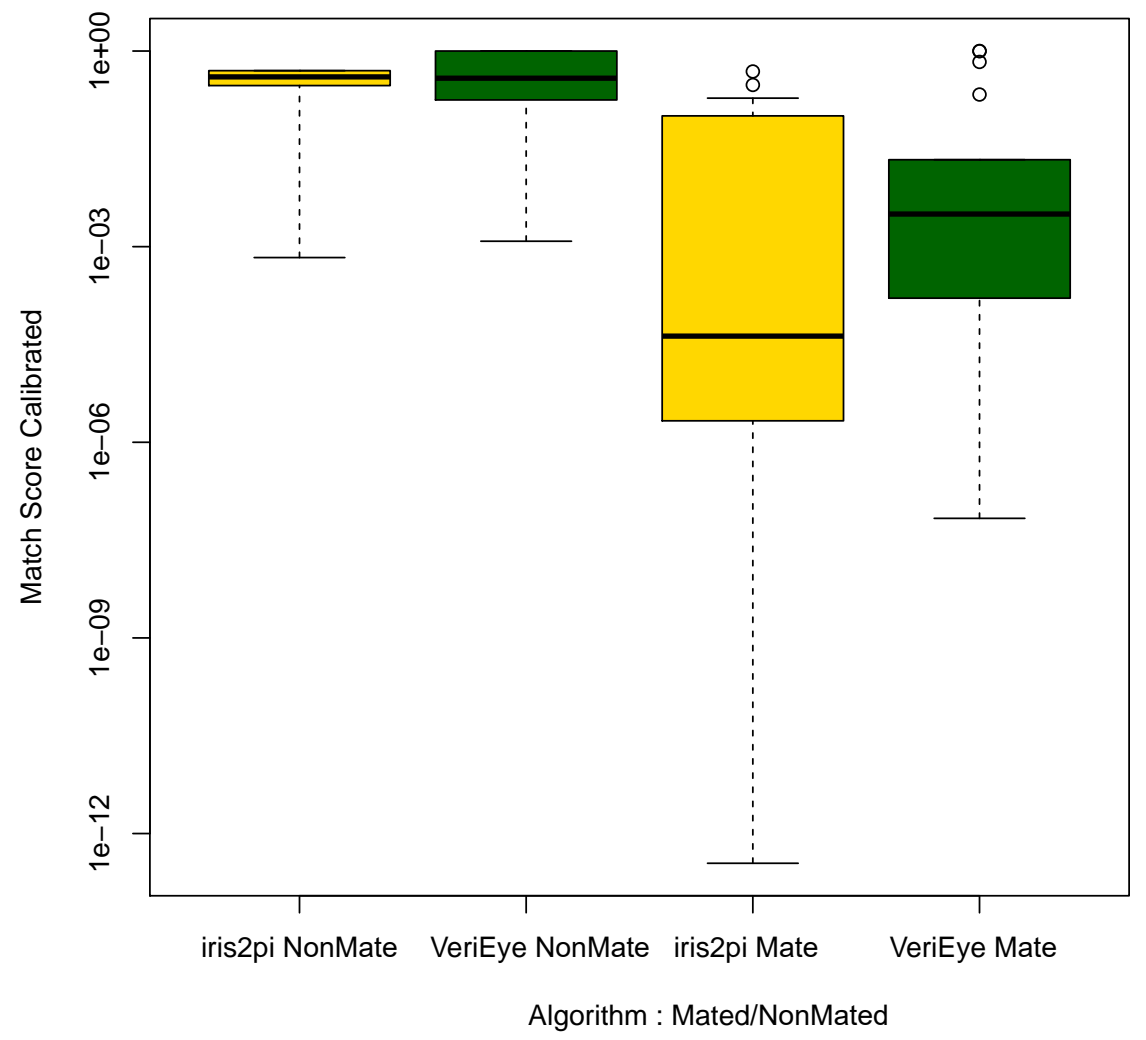

Fig. 8. (Box-plot by algorithm and mated/non-mated of match scores converted to a common (FMR) scale. 


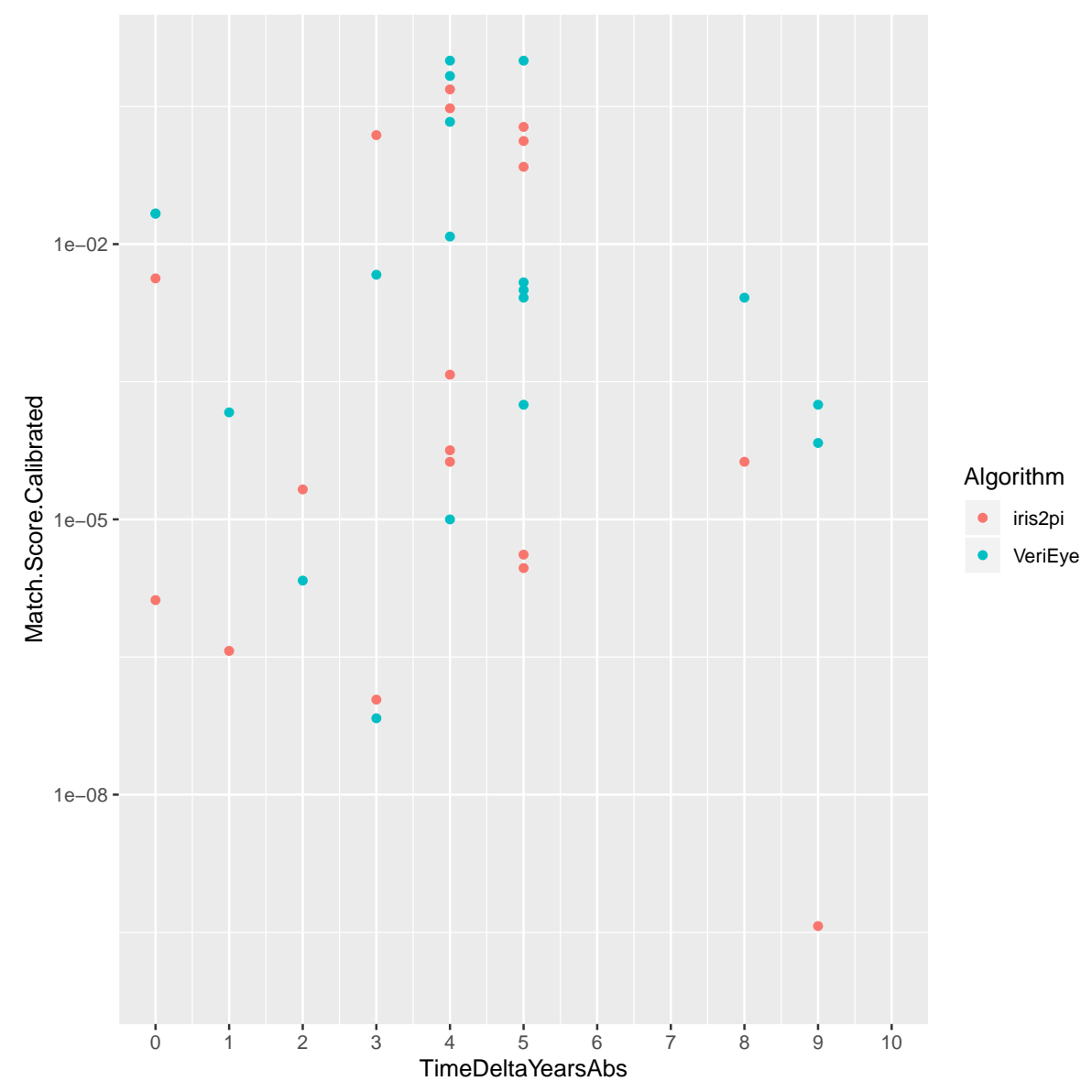

Fig. 9. Plot of match score on FMR scale for mated images vs. time (years) between image collection by algorithm. 


\section{Conclusions}

We expanded on the Afghan Girl study by Daugman using gray-scale photographs of 4 subjects taken every year for 40 years; we extracted iris images from the photographs and generated match scores using two commercial iris recognition algorithms. We developed criteria for making objective decisions about the utility of the extracted iris images and restricted the analysis of the mated pairs to those satisfying the criteria. The resulting match scores demonstrate matches at thresholds that correspond to FMR of 0.01 or better over periods of up to 9 years.

These are the initial results; optimization of the process may improve the results.

\section{Acknowledgments}

Our thanks to the Nixon family and to the Fraenkel Gallery for agreeing to license the high resolution images for use in our research and for permission to use the low resolution examples that illustrate the paper.

The Brown Sisters images are @Nicholas Nixon, and courtesy Fraenkel Gallery, San Francisco.

Our thanks to Mei Ngan and Bethany Retton for helpful discussions that improved the quality and readability of the paper.

Our thanks to the staff supporting the Biometrics Research Laboratory for providing the resources that enable our research. 


\section{References}

[1] Matey JR, Matey JR, Quinn GW, Grother PJ (2019) IREX Validation Dataset 2019. NIST, Technical Report TN-2058.

[2] Nixon N (2014) The Brown Sisters: Forty Years (Museum of Modern Art.), .

[3] Quinn GW, Matey J, Tabassi E, Grother PJ (2014) IREX V: Guidance for iris image collection. National Institute of Standards and Technology, Technical Report NISTIR-8013. https://doi.org/10.6028/NIST.IR.8013. URL https://www.nist. gov/publications/irex-v-guidance-iris-image-collection

[4] Quinn GW, Quinn GW, Grother P, Matey J (2019) IREX IX Part Two: Multispectral Iris Recognition. NIST, Technical report. URL www.nist.gov/itl/iad/image-group/ irex-ix.

[5] Miot HA, et al. (2009) Comparative evaluation of oculometric variables in graves' ophthalmopathy. Clinics 64(9):885-889.

[6] Daugman J (2001) Iris recognition. American scientist 89(4):326-333. URL https $/ /$ www.americanscientist.org/issues/issue.aspx $? \mathrm{id}=737 \& \mathrm{y}=2001 \& \mathrm{no}=4 \&$ content $=$ true $\&$ page $=4 \& \operatorname{css}=$ print .

[7] Daugman J (2004) How iris recognition works. Circuits and Systems for Video Technology, IEEE Transactions on 14(1):21-30. URL http://ieeexplore.ieee.org/xpls/ abs_all.jsp?arnumber=1262028.

[8] Daugman J (2006) Probing the uniqueness and randomness of iriscodes: Results from 200 billion iris pair comparisons. Proceedings of the IEEE 94(11):1927-1935.

[9] Daugman J (2016) Evolving methods in iris recognition. IEEE International Conference on Biometrics: Theory, Applications, and Systems, . URL http://www.cse.nd. edu/BTAS_07/John_Daugman_BTAS.pdf.

[10] Masek L (2003) Recognition of human iris patterns for biometric identification. Ph.D. thesis. Master's Thesis, Univesity of Western Australia, .

[11] Daugman J (2007) New methods in iris recognition. IEEE Trans Systems, Man, Cybernetics $B$ 37(5):1167-1175.

[12] Matey JR, Broussard R, Kennell L (2010) Iris image segmentation and sub-optimal images. Image and Vision Computing 28(2):215-222. 
[13] Neurotechnology (2017) MegaMatcher 10.0, VeriFinger 10.0, VeriLook 10.0, VeriEye 10.0 and VeriSpeak 10.0 SDK, Developers Manual, .

[14] Grother PJ, Matey JR, Tabassi E, Quinn GW, Chumakov M (2013) IREX VITemporal stability of iris recognition accuracy. NIST, Technical report. URL doi. org/10.6028/NIST.IR.7948.

[15] Das PD, Holsopple L, Rissacher D, Schuckers M, Schuckers S (2020) Iris recognition performance in children over time: A longitudinal study. Submitted to The 2nd Workshop on Demographic Variation in the Performance of Biometric System, WACV 2020 .

[16] Strochlic N (2017) Famed 'Afghan Girl' Finally Gets a Home. National Geographic URL https://www.nationalgeographic.com/news/2017/12/ afghan-girl-home-afghanistan/.

[17] Daugman J (2004) How the Afghan Girl was Identified by Her Iris Patterns, . URL https://www.cl.cam.ac.uk/ jgd1000/afghan.html. 